\title{
VISUOTOPIC ORGANIZATION OF THE PRELUNATE GYRUS IN RHESUS MONKEY ${ }^{1}$
}

\author{
W. M. MAGUIRE ${ }^{*} \ddagger$ AND J. S. BAIZER ${ }^{2}$

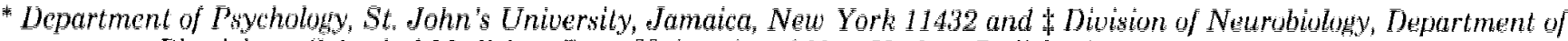 \\ Physiology, School of Medicine, State University of Nèw York at Buffalo, Amherst, New York 14226
}

Received August 15, 1983; Revised December 27, 1983; Accepted January 18, 1984

\begin{abstract}
We have examined topographic organization of the prelunate gyrus and adjacent cortex buried in the lunate and superior temporal sulci. We recorded from cortex of awake rhesus monkeys performing a fixation task. Multiunit receptive fields were mapped with small, stationary spots of light to determine borders and points of strongest driving or "activity centers" of the fields. We found evidence for several distinct subdivisions of this cortex. A representation of the vertical meridian runs across the gyrus, and two crude topographic representations of the central $30^{\circ}$ of the lower quadrant, the posteromedial and anterolateral areas (area PM and area AL), share this representation of the meridian. Area AL extends from the prelunate gyrus into the posterior bank of the superior temporal sulcus; it is separated from the M'T area by a narrow strip of cortex. Area PM occupies part of the prelunate gyrus and extends into the anterior bank of the lunate sulcus. Receptive field size in both AL and PM is an increasing function of eccentricity and is similar for the two areas. Medial to areas PM and AL on the prelunate gyrus is another cortical region with qualitatively different topographic organization.
\end{abstract}

Prestriate cortex has traditionally been divided into two zones, areas 18 and 19 , based upon the cytoarchitectonic studies of Brodmann. In recent years, the use of anatomical and physiological techniques has resulted in a more complex picture of prestriate cortex. In a number of mammals, including cat, owl monkey, and rhesus monkey, prestriate cortex contains a multiplicity of topographic maps of the visual field (see Van Essen, 1979, for review). These maps form a mosaic of visual areas, or representations of visual space. It has been suggested that these multiple visual areas represent functional specializations in the analysis of visual information, and differences in receptive field size, magnification factor, and stimulus selectivity have been found among the various areas (Zeki, 1973, 1977, 1978b; Baizer et al., 1977; Van Fssen, 1979; Baker et al., 1981; Baizer, 1982). The overall topographic organization of many of these areas

\footnotetext{
${ }^{1}$ This work was supported by National Institutes of Health Grants RO1 EY02230 and T32 EY07019. We thank D. Bender, S. Udin, and J. Mcllwain for critical reading of the manuscript, Diana Bozian, Brissen Elmer, and Aviva Abosch for technical assistance, Jim Stamos for illustrations, S. Udin, for use of computer graphics facilities, Avi Snyder for PDP-11 software, Julie Lakatos for photography, and Susan Wolf, Linda Smith, Denise Bilancini, and Jean Seiler for secretarial assistance.

${ }^{2}$ To whom correspondence should be addressed.
}

has been described, and this organization is particularly well understood in the owl monkey (Allman and Kaas, 1971, 1974a, b, 1975, 1976).

The major work describing the organization of prestriate cortex in the rhesus monkey has been that of Zeki (1969, 1970, 1971, 1973, 1977, 1978a, b, c; Van Essen and Zeki, 1978). Using both physiological and anatomical techniques, he has argued for the existence of a number of distinct visual areas in prestriate cortex, including V2, $\mathrm{V} 3, \mathrm{~V} 3 \mathrm{~A}$, an area in the posterior bank of the superior temporal sulcus which is now generally called MT (Gattass and Gross, 1981; Van Essen et al., 1981), and V4 or the fourth visual complex. The topographic organization of three of the areas, V2, V3, and MT, is fairly well understood (Van Essen, 1979; Gattass and Gross, 1981; Gattass et al., 1981; Van Essen et al., 1981). However, neither V3A nor the fourth visual complex have been completely mapped, and their full extent and boundaries are unknown (Van Essen and Zeki, 1978). V3A contains at least one representation of both upper and lower quadrants but may contain a double representation of central vision (Van Essen and Zeki, 1978). Van Essen and Zeki (1978) found electrophysiological evidence for topographic order in V4. Receptive fields were large, but still smaller than a quadrant, and fields recorded at neighboring sites tended to be in neighboring parts of the visual field. However, Van Essen and Zeki (1978) 
also found evidence for complexity in the representation, since some parts of the visual field seemed to be multiply represented. They concluded that V4 probably does not contain a single, orderly representation of either the quadrant or the hemifield.

In the present study we recorded from single units and multiunits on the prelunate gyrus and surrounding buried cortex in the rhesus monkey over an area partially coextensive with the "V4 complex." Receptive fields are quite large in this area and show scatter proportional to their size. It is very difficult to see topography if one relies solely upon conventional mapping techniques which simply analyze the progression of receptive fields along single penetrations, or between neighboring penetrations. We have thus developed a number of techniques for revealing the gross topography of this visual cortex. Our data suggest that the area contains several subdivisions with different degrees of topographic regularity. Brief reports of some of these results have appeared (Maguire and Baizer, 1981; Baizer and Maguire, 1983).

\section{Materials and Methods}

\section{Behavioral and recording procedures}

Recordings were made in awake, behaving monkeys, to eliminate effects of anesthesia and to minimize variability in responses due to variability in the level of arousal of the animal. The methods have been described in detail previously (Maguire and Baizer, 1982). Briefly, animals were first trained on the fixation task developed by Wurtz (1969). After pretraining, they were anesthetized and, under aseptic conditions, implanted with head restraint bolts and silver-silver chloride pellet electrodes for eye movement recording. A stainless steel microdrive base was implanted, centered approximately at stereotaxic coordinates $10 \mathrm{P}, 20 \mathrm{~L}$. The bolts provided the base for a headholder, which was permanently attached to the head. During recording sessions, the head was immobilized and the activity of single cells and small groups of cells was recorded with glass-insulated platinum-iridium microelectrodes with exposed tips of 10 to $20 \mu \mathrm{m}$. Recordings of eye movements were displayed on an oscilloscope and sent to a PDP 11-34 computer. Signals from the microelectrode were amplified and sent to a timewindow discriminator, displayed on an oscilloscope, and sent to an audio monitor. Output pulses from the discriminator were sent to the computer. Behavioral control and data analysis were accomplished by the computer. The computer also constructed rasters and histograms which were displayed on-line. Only trials during which the monkey maintained fixation were used in the data analysis.

\section{Visual stimulation}

Visual stimuli were presented automatically during fixation periods. Monkeys sat at a distance of from 40 to $114 \mathrm{~cm}$ from a large Polacoat screen. Background luminance was 0.15 foot-lambert. Stimuli were presented through a Leitz projector fitted with a rectangular diaphragm which permitted focusing of rectangular stimuli of different lengths, widths, and orientations on the screen. Shutters controlled stimulus onset and offset; narrow band color filters (Melles Griot) and Wratten neutral density filters could be inserted in the light path to vary stimulus color and luminance. Stimulus position and movement were controlled with an X-Y mirror system. The image projected on the screen was intercepted by a beam splitter and focused on a table to permit drawing of receptive fields.

\section{Experimental protocol}

At the beginning of each penetration, the electrode was lowered until cells were first encountered. The visual field was then searched with fairly large stimuli until an effective region was found. We then attempted to obtain multiunit responses from this region using small, rectangular, flashed spots. Generally such a stimulus was adequate, but if consistent responses could not be obtained, we varied color, orientation, size, shape, or luminance of the stimulus, or allowed it to move along a short trajectory until adequate multiunit responses were elicited. Rarely did this procedure fail to allow us to plot the multiunit receptive field; when it did, the electrode was moved slightly and we began again.

We first determined the full extent and boundaries of multiunit fields as well as the approximate location of the activity center of the fields. The multiunit receptive field was defined as the entire extent of the visual field where small stimuli elicited consistent responses. The "activity center," or point of strongest driving of the receptive fields, was defined as the location in the receptive field where the stimulus used to plot the whole field evoked the strongest response. In order to locate activity centers with precision, we used computer-constructed raster displays which counted and displayed the number of spikes in the response. Figure 1 illustrates a plot of a typical multiunit receptive field, showing borders and activity center. Once the multiunit field was mapped, the electrode was lowered in 0.5 - or 1 -mm steps on penetrations into the lunate or superior temporal sulcus, or a new penetration was made. Occasionally, receptive fields of single cells were mapped to determine receptive field boundaries and stimulus specificities. Generally, a single penetration into the lunate or the superior temporal sulcus, or two to three surface penetrations were made in a single experimental session. On some deep penetrations into the sulci, small electrolytic lesions were made to aid in later anatomical reconstruction.

We used a polar coordinate system to describe the location of activity centers in visual space. Each point was defined by two numbers, eccentricity and angle. The lower vertical meridian was assigned an angle of $0^{\circ}$, and the contralateral horizontal meridian was assigned an angle of $90^{\circ}$. Measurements made on-line were subsequently corrected for distance of the monkey from the screen, and for the fact that the screen was a flat rather than a spherical surface.

\section{Histology}

Upon conclusion of the recording, the animal was deeply anesthetized and perfused with saline and then formalin. The head was removed, and pins were inserted into the brain using the microdrive. The brain was then 


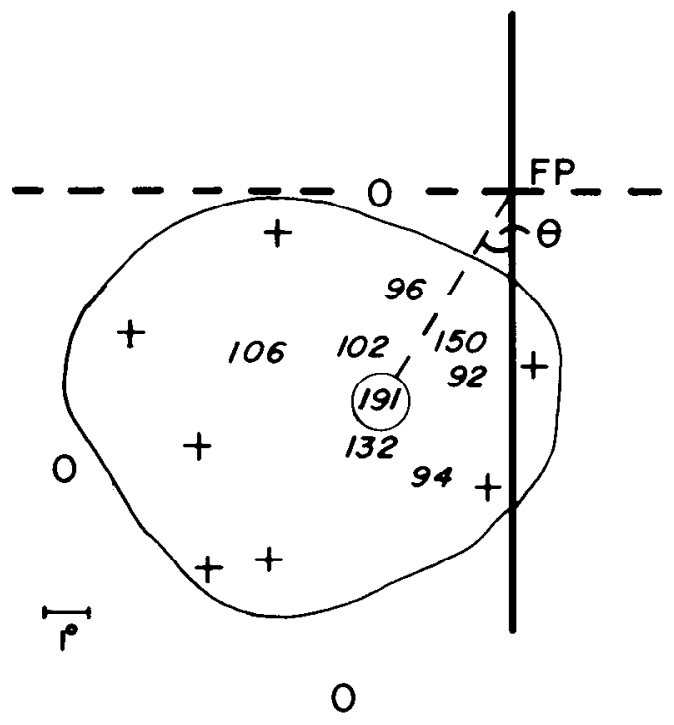

Figure 1. Plot of a typical multiunit field mapped with stationary flashed stimuli. The solid vertical line indicates the vertical meridian; the broken horizontal line indicates the horizontal meridian. FP shows the location of the fixation point. On-responses are indicated by $+; 0$ indicates no response. Numbers show the total number of action potentials elicited by three presentations of a small flashed spot at the positions indicated. The activity center, indicated as the circled number, was at eccentricity $5 \frac{1}{1} 2^{\circ}, \theta=35^{\circ}$.

removed. Two pins were inserted into the brain in the horizontal plane to aid in the alignment of serial sections. Forty-micrometer frozen sections were cut in the parasagittal plane and stained with cresyl violet. Electrode penetrations were reconstructed from microscopic examination of sections. Unmarked penetrations were reconstructed on the basis of their positions in the recording chamber relative to marked tracks and marking pins. We were able to find all marking lesions.

\section{Construction of unfolded map}

To facilitate analysis of visuotopic organization, we constructed an unfolded map of the relevant part of the hemisphere. Since we were concerned with a relatively small expanse of cortex, we were able to use a simple and straightforward unfolding of the cortex (as in Hubel and Wiesel, 1972) rather than the more complex method developed by Van Essen and Maunsell (1980) for unfolding large areas. Our procedure is illustrated in Figure 2, which shows the unfolding of the lunate sulcus, prelunate gyrus, and posterior bank of the superior temporal sulcus. We first calculated a shrinkage factor of $20 \%$, based on the measured distance between pins before and after processing of the tissue. We assumed shrinkage was isotropic and corrected all measurements accordingly. We took a series of parasagittal sections spaced $0.5 \mathrm{~mm}$ apart (Fig. $2 B$ shows three representative sections). We traced layer 4 in each section (Fig. $2 B$, dashed lines with arrowheads). We chose as a zero point the posterior crest of the prelunate gyrus (heavy line normal to the surface in sections in Fig. $2 B$ ) and for each section measured distances along the traced lines anterior and posterior to that point. Lines from all sections (Fig. $2 C$, dashed lines with arrowheads) were then drawn onto a two-dimen- sional plot with a coordinate system showing the mediolateral stereotaxic location of the sections (Fig. $2 \mathrm{C}, \mathrm{Y}$ axis) and distances in millimeters posterior and anterior to the zero point (Fig. $2 C, X$-axis).

\section{Results}

\section{Analysis of topography: Sector maps}

Our analysis is based on data from 358 recording sites from 174 penetrations in five hemispheres of three animals. The cortex of the prelunate gyrus is quite visually responsive, although it is harder to elicit responses from more medial parts of the gyrus. We analyzed visual topography by looking at the cortical representation of parts, or sectors, of the visual field. Figure 3 illustrates our analysis for one hemisphere.

We divided the lower field into sectors by first drawing radii at $30^{\circ}$ and $60^{\circ}$ from the lower vertical meridian (Fig. $3 A$ ). "A" sectors border the vertical meridian, "C" sectors border the horizontal meridian, and " $B$ " sectors lie in between. The field was further divided by drawing arcs at $3^{\circ}, 5^{\circ}, 10^{\circ}$, and $20^{\circ}$ eccentricity, a spacing that reflects an approximate correction for cortical magnification factor. The upper field was divided in half by a radius at $45^{\circ}$ from the upper vertical meridian. For each recording site, the activity center was assigned to the appropriate sector, and recording sites with their sector assignments were plotted on the unfolded map of the cortex (Fig. $3 B$ ). We simplified this plot by drawing a convex area that contained all neighboring recording sites with field centers falling within the same sector (Fig. 3C). Finally, we used the sector map to construct a visuotopic summary map showing vertical meridian (Fig. $3 D$, solid line), horizontal meridian (Fig. $3 D$, dashed line), and lines of isoeccentricity (Fig. $3 D$, dotted lines).

Construction of visuotopic summary maps involved somewhat arbitrary decisions about placements of lines for the meridia. Where a meridian representation fell between two mirror image maps, the meridian was drawn by bisecting the region corresponding to the appropriate sectors, although this could lead to error in meridian placement if magnification factor for the two representations differed. Where upper field sites were found adjacent to lower field sites, the horizontal meridian was drawn to fall between upper (sectors D and E) and lower (sector C) field representations. Lines of isoeccentricity were placed between consecutively numbered sectors.

Figures $4 A$ and $5 A$ show sector maps for two additional hemispheres, and Figures $4 B$ and $5 B$ show the visuotopic summary maps for those hemispheres. The data shown in these figures were confirmed by less extensive recordings in two additional hemispheres.

\section{Topography of the prelunate gyrus: Areas AL and PM}

The most important visuotopic landmark, and one present in all hemispheres studied, was a representation of the vertical meridian. On the prelunate gyrus, just a few millimeters above the intersection with the lateral fissure, multiunit fields have activity centers at eccentricities of 15 to $25^{\circ}$ near the lower vertical meridian (sector A4 representation in Figs. 3 to 5). Activity centers cluster around the vertical meridian, and fields can ex- 
tend as far as $30^{\circ}$ into the ipsilateral field. Fields continue to cluster around the vertical meridian but move toward the fovea as one moves laterally onto and across the gyrus. The meridian representation continues into the anterior bank of the lunate sulcus just below the level of the lateral fissure. At the posterior edge of the gyrus, fields are at an eccentricity of 5 to $7^{\circ}$ (representation of sector A3 in Figs. 3 to 5). The meridian representation moves down the anterior bank in a generally ventral direction, and fields become increasingly foveal as one moves into the sulcus (representation of sectors $\mathrm{A} 1$ and $\mathrm{A} 2$ in Fig. 4, A2 in Fig. 3, and $\mathrm{A} 1$ and $\mathrm{A} 2$ in Fig. 5). Although the meridian representation was present in all hemispheres, its location relative to geographic landmarks varied from hemisphere to hemisphere (cf. Figs. 3,4 , and 5 ).

Posterior and medial to this representation of the meridian, we have found one representation of the lower contralateral quadrant which we call area PM. Anterior and lateral to the vertical meridian, there is a second representation of the quadrant which we call area AL. Both areas appear to be organized topographically, including approximately the central $30^{\circ}$ of the quadrant, and they are roughly mirror images of each other.

Area PM is shown in Figures $3 D$ and $4 B$. It occupies part of the prelunate gyrus and extends into the anterior bank of the lunate sulcus. Dorsomedially, area PM is bordered by a representation of the horizontal meridian. This meridian representation runs generally parallel to the representation of the vertical meridian at a distance of 3 to $4 \mathrm{~mm}$ from it. Thus, peripheral fields clustering around the horizontal meridian are found posterior to the peripheral vertical meridian on the prelunate gyrus (sectors $\mathrm{C} 3$ and C4, Figs. 3 and 4; C3, Fig. 5); fields

A.

\section{B. Parosagittal Sections}

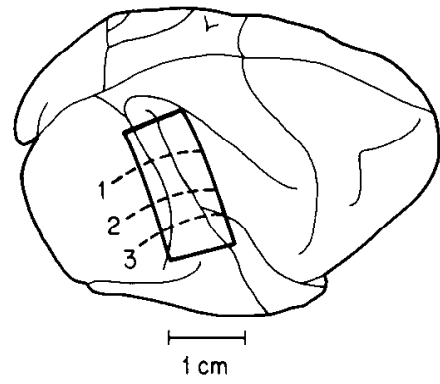

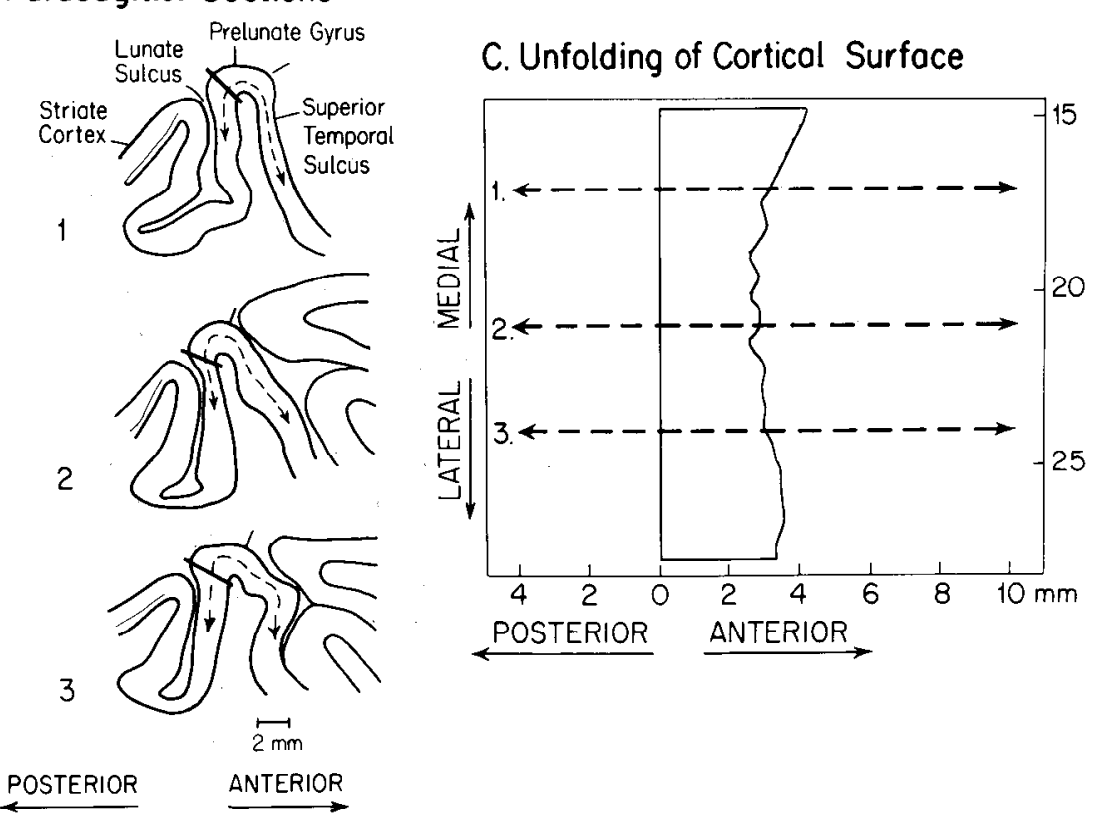

Figure 2. Procedure for unfolding the cortical surface and resultant twodimensional map. A, Dorsolateral view of the rhesus monkey brain. The region of the prelunate gyrus studied is enclosed in the solid rectangle. $B$, Three parasagittal sections through the prelunate gyrus and lunate and superior temporal sulci at levels 1,2 , and 3 indicated by the dashed lines in $A$. The heavy line normal to the cortical surface indicates the zero landmark used for aligning serial sections. Tic marks indicate the anterior crest of the prelunate gyrus. Dashed lines with arrowheads represent tracings of layer $4 . C$, Unfolded representation based on such sections. This is the same hemisphere as in Figure 4. The $x$ axis indicates millimeters from the zero point, the posterior crest of the gyrus. The $y$ axis indicates stereotaxic medial-lateral coordinates. The jagged line represents the anterior crest of the gyrus. 

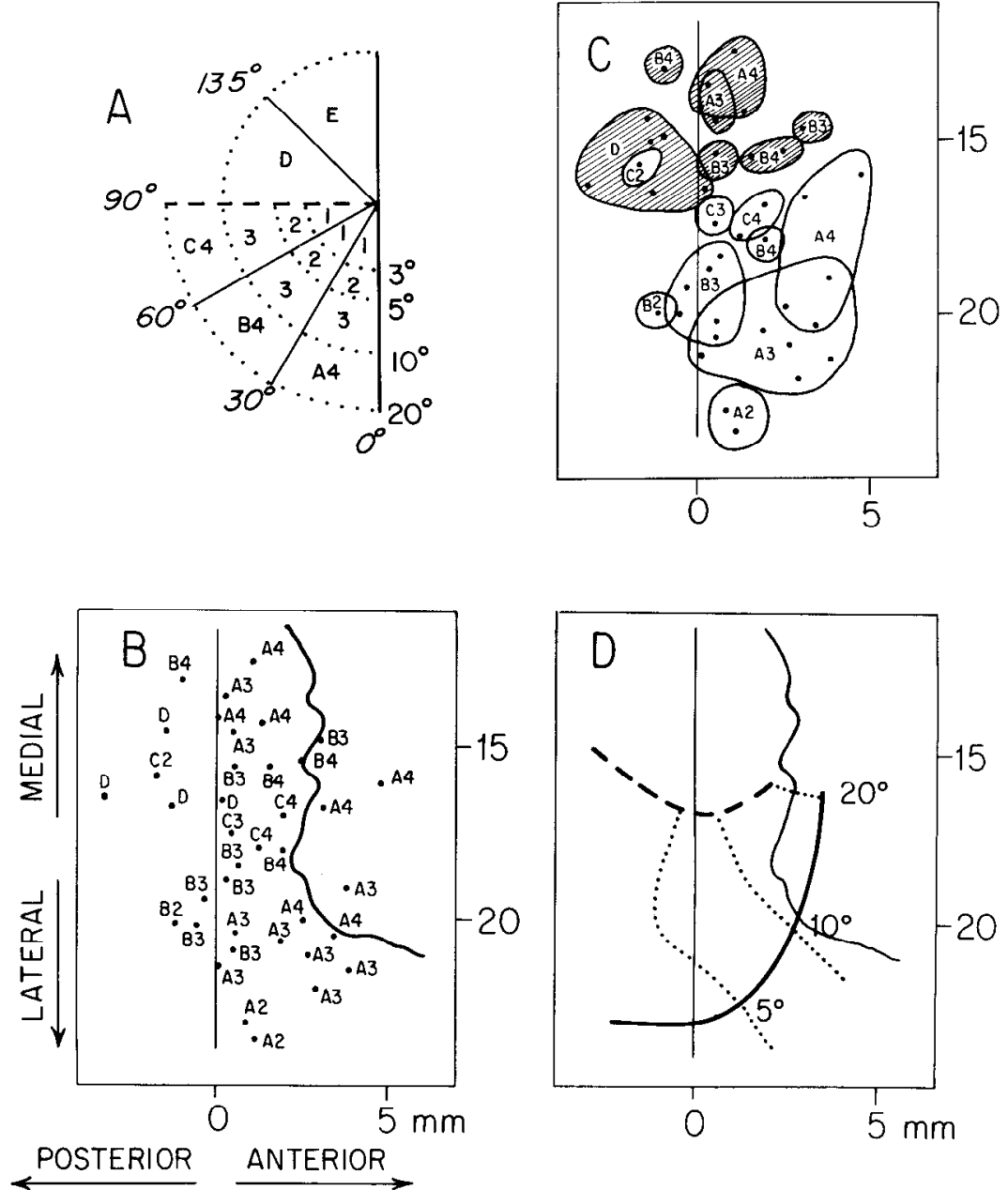

Figure 3. $A$, Division of the visual hemifield into 14 sectors. $B$, Unfolded map of hemisphere with locations of recording sites indicated by dots. The multiunit field activity center at each site was assigned to the appropriate visual field sector, and recording sites were labeled accordingly. $C$, Neighboring recording sites with the same sector assignments are enclosed by solid lines. Shaded sectors show cortex dorsal to the two mapped visual areas. $D$, Visuotopic summary map of this hemisphere.

become more central as the horizontal meridian moves laterally, and finally ventrally, into the anterior bank of the lunate sulcus.

Area $A L$ is shown in Figures $4 B$ and $5 B$. This area occupies the anterior part of the prelunate gyrus and extends well into the posterior bank of the superior temporal sulcus. Area AL is bordered anteriorly by a representation of the horizontal meridian, which lies entirely buried within the posterior bank of the superior temporal sulcus. Peripheral sites on the horizontal meridian are found anteromedially (Fig. 4, C4 at about stereotaxic 17 lateral and $7 \mathrm{~mm}$ anterior to 0 ). Parafoveal sites are located more laterally (Fig. 4, C3, C2, and C1 sectors at about 20 lateral and $7 \mathrm{~mm}$ anterior to 0 ). Figure 6 shows the typical progression of receptive fields on three representative penetrations down the posterior bank of the superior temporal sulcus. In penetrations $a$ and $b$, the electrode begins in area $\mathrm{AL}$, and fields move to the horizontal meridian. In penetration $c$, the electrode begins in PM, and fields move first toward the vertical meridian (sites 1,2, and 3) and then, as the electrode moves through AL, the fields shift back toward the horizontal meridian.

The sector maps clearly suggest the presence of topographic order in AL and PM. Neighboring recording sites have activity centers in the same or adjacent visual field sectors. There is an orderly progression of sector representations across the cortex in both areas. A sectors lie next to B sectors; B sectors lie next to C sectors. Similarly, consecutively numbered sectors are adjacent. Evidence for topographic order was least clear for area $\mathrm{AL}$ in the hemisphere shown in Figure 5; activity centers for some recording sites lay in distant sectors. Nevertheless, the overall progression of sectors across the cortex is still discernible. In order to estimate how well the summary maps account for our data, we determined the proportion of recording sites whose activity centers fell into the visual field sector, or its neighbor, indicated by the summary map. For the maps shown in Figures 3, 4, and 5, the proportions were: $100 \%, 91 \%$, and $80 \%$. However, 
Figures 3, 4, and 5 all show overlap in the sector representations, suggesting that areas AL and PM lack much of the topographic precision seen at earlier stages of the visual system.

\section{Cortex bordering areas $P M$ and $A L$}

Areas PM and AL are bordered medially by a zone of cortex which appeared qualitatively different in its organization. We have indicated this zone by the shaded sectors in Figures 3, 4, and 5. The posterior half of this zone lies on the prelunate gyrus and extends into the anterior bank of the lunate sulcus. Multiunit fields are centered in the upper visual field (sectors $D$ and $E$, Figs. 3 and 4). Eccentricities are about the same as in the neighboring parts of area PM. Some sites had fields near the horizontal meridian; others had fields near the upper vertical meridian, often extending well into the ipsilateral hemifield. However, the location of multiunit fields did not suggest a simple, orderly representation of the upper quadrant, analogous to the lower quadrant representation in area $\mathrm{PM}$.

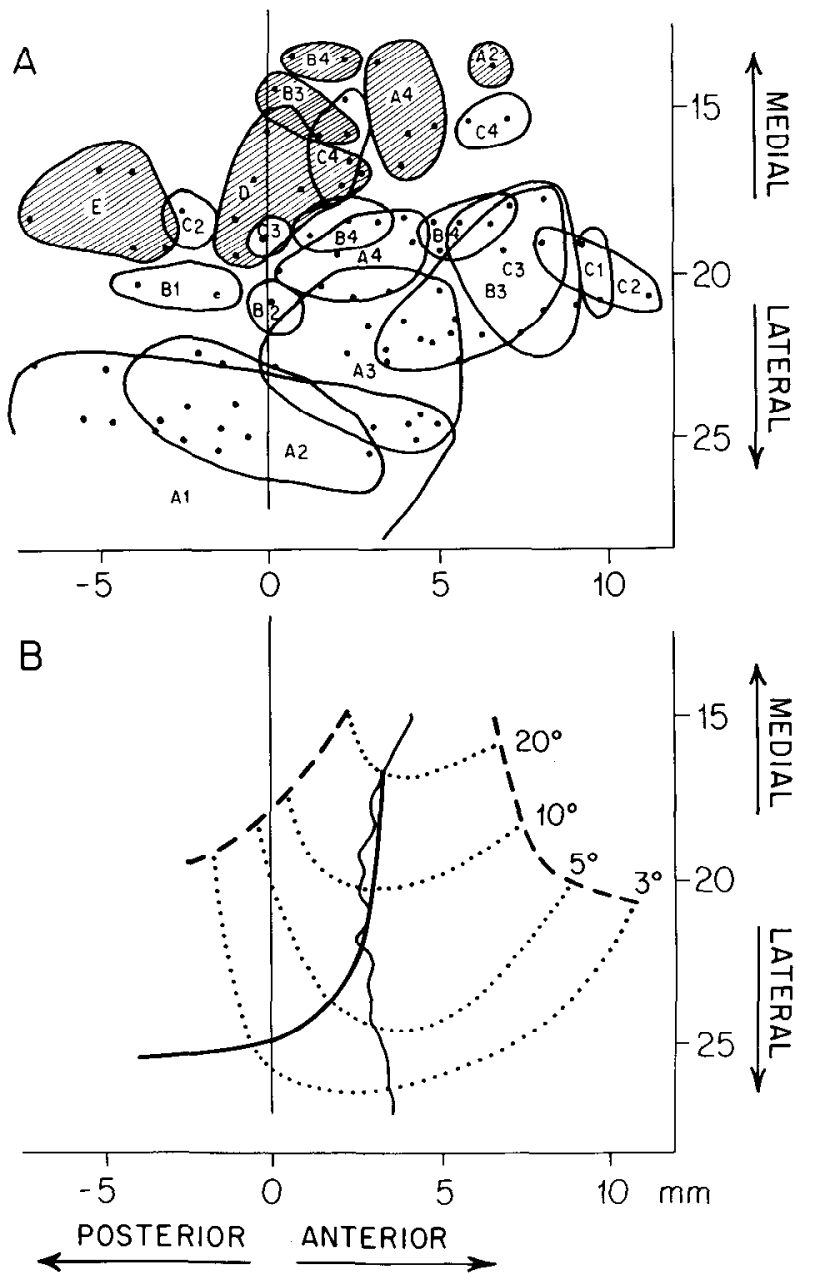

Figure 4. Sector map $(A)$ and visuotopic summary map $(B)$ for a second hemisphere. This hemisphere shows the relation of the two visual areas to each other. Shaded sectors are in cortex dorsal to those areas.
The anterior half of this border zone (Figs. 3 to 5 , shaded sectors $A, B$, and $C$ ) extends from the prelunate gyrus into the superior temporal sulcus. Multiunit fields here are much larger than in areas PM and AL, and they may cover extensive areas of the visual field including the fovea. Fields appear randomly scattered but cluster in the lower visual field near the vertical meridian (Fig. $3 C$, shaded sectors $B A, B 3$, and $A 4$; Fig. 4 , shaded sectors
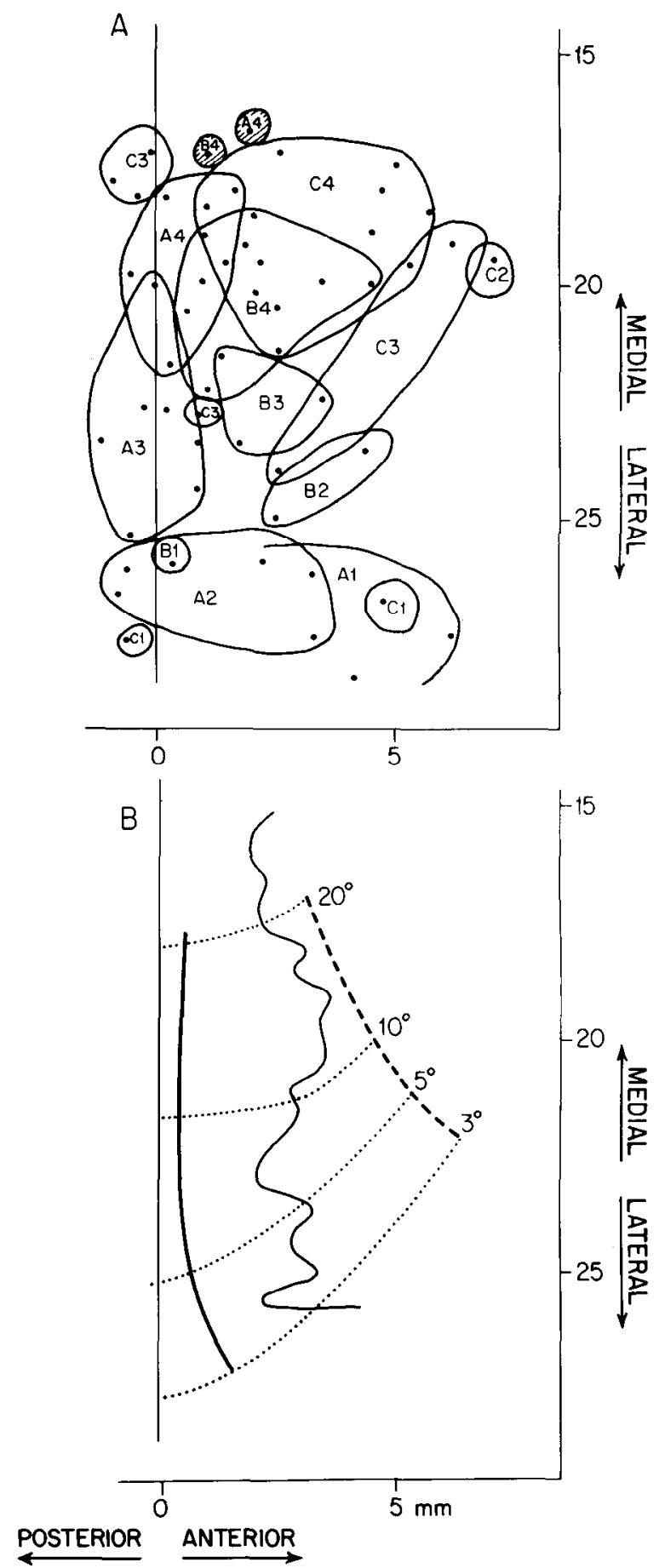

Figure 5. Sector map $(A)$ and visuotopic summary map $(B)$ for a third hemisphere. This hemisphere gave the most information about the anterolateral area and its borders. Shaded sectors are in cortex dorsal to this area. 
$A 4, B 3$, and $B 4)$. We have been unable to find any clear transition between the posterior upper visual field sites and these more anterior sites. Medial to this border zone of cortex, cells become extremely unresponsive to the simple visual stimuli we have used, and we were unable to discover much about their visual receptive field properties.

Area AL is bordered anteriorly by a narrow strip of

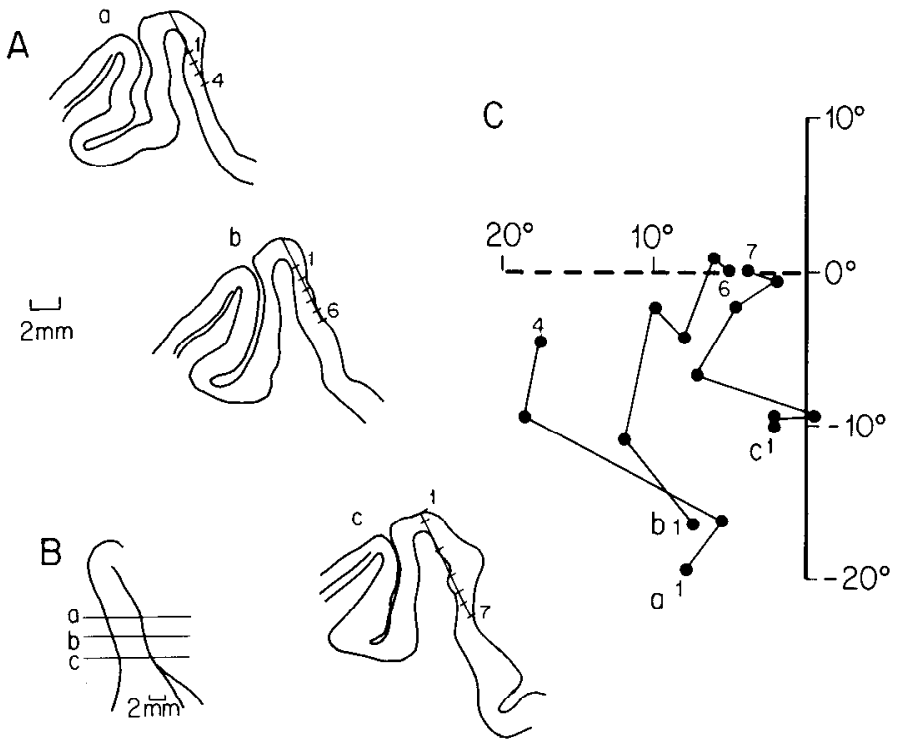

Figure 6. $A$, Three parasagittal sections through prelunate gyrus at the levels shown in $B$, showing three penetrations and recording sites. $C$, Multiunit field activity centers for each recording site shown in $A$. cortex in the superior temporal sulcus. The strip lies contiguous with, and posterior to, area MT which itself occupies cortex deep in the posterior bank of the sulcus (Gattass and Gross, 1981; Van Essen et al., 1981). As an electrode moves down the superior temporal sulcus beyond the horizontal meridian representation in AL, multiunit fields continue to be driven by flashed spots of light, but often responses are dramatically stronger if the stimuli are moved rather than flashed. These responses are not directionally selective, but can be elicited by a spot properly positioned and moved in any direction. As the electrode is moved through this zone of motionsensitive cortex, field positions move rapidly but continuously toward the vertical meridian (see Fig. 7). Multiunit fields centered on or near the vertical meridian are encountered after the electrode has moved a rather short distance ( 1 or $2 \mathrm{~mm}$ in the more medial penetrations). At the vertical meridian, there is a second change in most multiunit field properties; they become dramatically direction sensitive. Given the position of fields on or near the vertical meridian and the presence of directional selectivity, it is likely that the electrode has entered MT at this point (cf. Gattass and Gross, 1981; Van Essen et al., 1981) and that MT and AL do not share a common border.

We did not fully explore the foveal representation of either area PM or AL. Furthermore, we have found it much more difficult to determine the ventral and lateral borders of PM and AL where the fields are increasingly foveal. As pointed out in the "Discussion," it is difficult, if not impossible, to establish boundaries of areas based on the location of vertical and horizontal meridian representations when multiunit fields are large and close to the fovea.
A
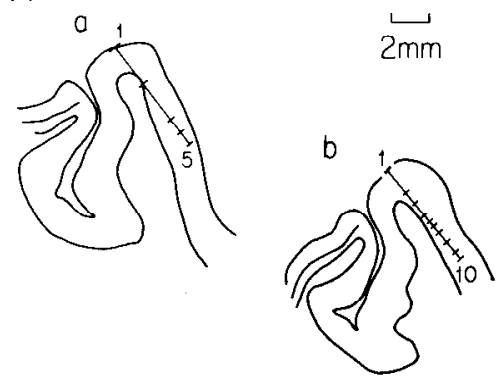

B

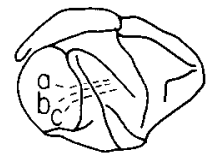

- AL

$\triangle$ TRANSITION CORTEX

O MT

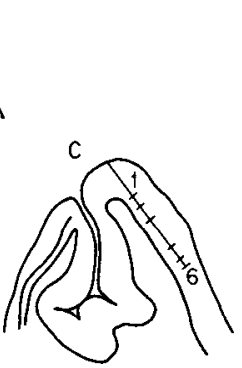

$\mathrm{C}$
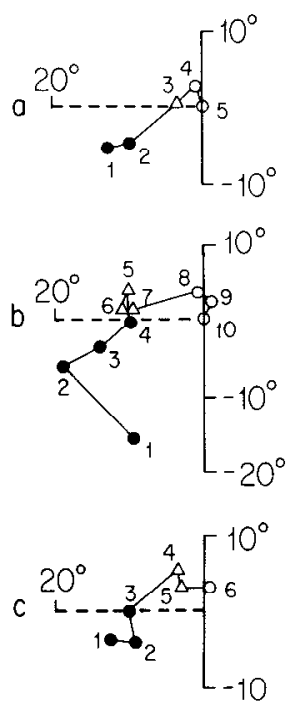

Figure 7. A, Three parasagittal sections through prelunate gyrus, showing threc penctrations $(a, b$, and $c)$ and recording sites along those penetrations. $B$, Location of sections on the prelunate gyrus. $C$, Locations of activity centers in the visual space are illustrated for each recording site. Solid circles represent sites in AL. Open triangles show sites that had movement sensitivity without directional selectivity and are in the transition cortex between $\mathrm{AL}$ and MT. Open circles mark recording sites where fields were directionally selective and identified as within MT. 


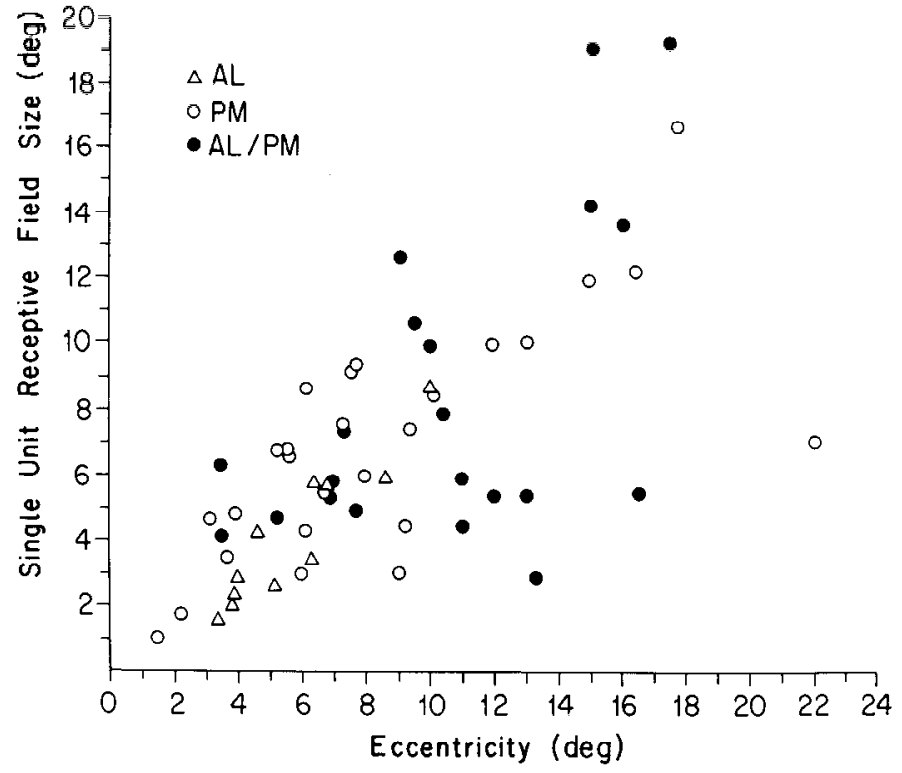

Figure 8. Single unit receptive field size as a function of eccentricity. Size is calculated as square root of area. Open triangles represent data from AL, open circles data from PM, and solid circles data from sites too close to the border to be reliably assigned to either area.

\section{Receptive field size and magnification factor}

Figure 8 shows the scatter plot of single cell receptive field size with eccentricity, and Figure 9 shows the same plot for multiunit fields. Fields in both PM and AL are about the same size and increase in approximately linear fashion with increased eccentricity. Since standard regression lines assume that variance is independent of eccentricity, and this clearly is not the case for our data, we fit a regression curve that minimizes variance after the scatter has been weighted in inverse proportion to eccentricity, that is, the line that minimizes: $\Sigma\left(\frac{s-\bar{s}}{e}\right)^{2}$, where $s$ is receptive field size and $e$ is eccentricity (see "Appendix A" for derivation). Using this method, the line describing single unit receptive field size (square root of area) as a function of eccentricity is $S=0.56 e+$ 1.79. For multiunit field size, $S=0.79 e+3.3$. As expected, multiunit fields are larger than single cell receptive fields, and they are considerably larger than those of V1 or V2. For multiunit fields, the slope of the line is comparable to the value of 0.91 for MT (Gattass and Gross, 1981).

We have calculated magnification factor for area $\mathrm{AL}$ in two hemispheres (Fig. 10). Magnification factor was estimated by choosing points from all penetration pairs whose separation was at least $1 \mathrm{~mm}$, but less than 2.5 $\mathrm{mm}$. As in any calculation of magnification factor, this is a somewhat arbitrary decision. Points very far from each other will yield poor estimates of magnification, since the points will often represent rather different parts of the visual field than the eccentricity at which the estimate of magnification factor will be defined. Points very close to each other will also yield poor estimates: as the true distance between receptive fields in the visual

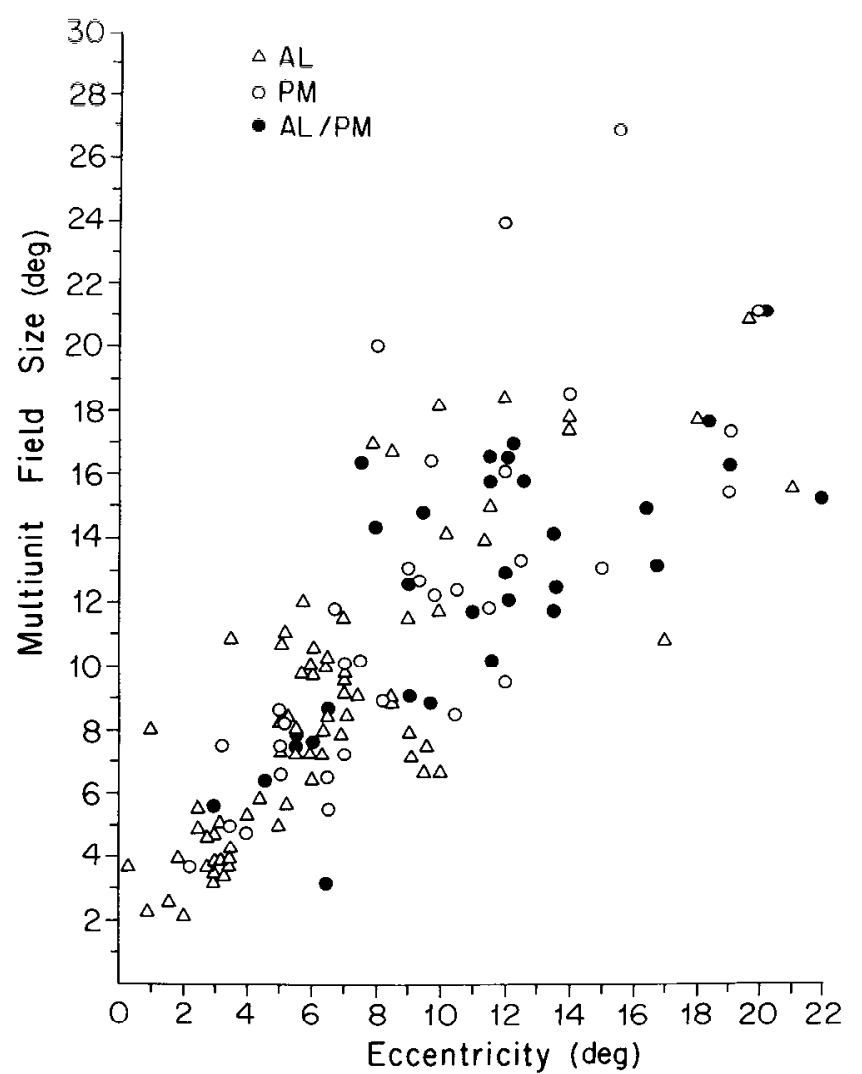

Figure 9. Multiunit field size as a function of eccentricity, using the same conventions as in Figure 8.

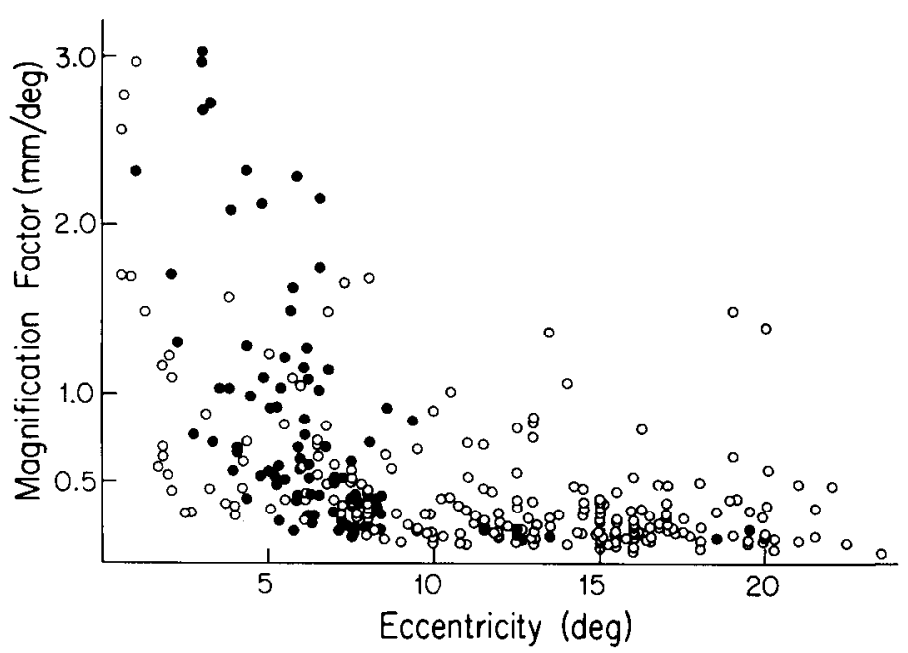

Figure 10. Magnification factor for points within AL from two hemispheres of two different monkeys (open and solid circles).

field gets smaller, the measured visual field separation increasingly reflects error variance in the mapping procedures, rather than the true receptive field separation. Thus, for increasingly close spacing there will be an increasingly severe and systematic miscalculation of magnification factor. We therefore chose points at an intermediate spacing. Figure 10 shows that magnification factor decreases with eccentricity and that data from the two hemispheres were similar. To compare magnification factor in AL with other areas, we fit the data with a 
power function and obtained $M=2.34 \times e^{-0.9}$ as the best estimate. A simple power function is clearly not the true function, since its value approaches infinity at very small eccentricities.

\section{Large receptive fields and topographic regularity}

We propose that areas AL and PM are organized topographically and have found that receptive fields in AL and PM are large. Mcllwain (1976) has pointed out that the presence of topography in visual areas with large receptive fields imposes certain spatial relations among the receptive fields of cells in a column. First, receptive fields of different sizes will not nest concentrically. Instead, they should nest eccentrically, with small fields displaced toward the fovea relative to the geometric center of the largest field. Although one cannot plot receptive fields of all cells in a column, one can record from several at once and plot the resulting multiunit field. Mcllwain's analysis suggests that the activity centers of multiunit fields should be displaced toward the fovea relative to the geometric centers by a predictable amount. (See "Appendix B" for a discussion of the predicted relation.) We have found that this is the case for our data. Figure $11 B$ shows the distribution of multiunit field activity centers relative to geometric centers for multiunit fields whose outlines we were able to determine with precision. In general, activity centers are displaced toward the fovea, and, although there is considerable variability, displacements do fall close to the predicted value (Fig. $11 B$, circled $X$ ).

A second prediction is that the receptive fields of all single cells of a column should include the activity center of the multiunit field derived from those cells. Again, it is not possible to test this prediction experimentally, but one can test a related prediction: receptive fields of single cells should overlap the activity center of a multiunit field recorded nearby. On a number of penetrations we were able to plot both a multiunit field and the receptive field of a single cell recorded less than $0.5 \mathrm{~mm}$ away. Using our regression curve for average receptive field size, we calculated the maximum separation between single unit receptive field centers and the associated
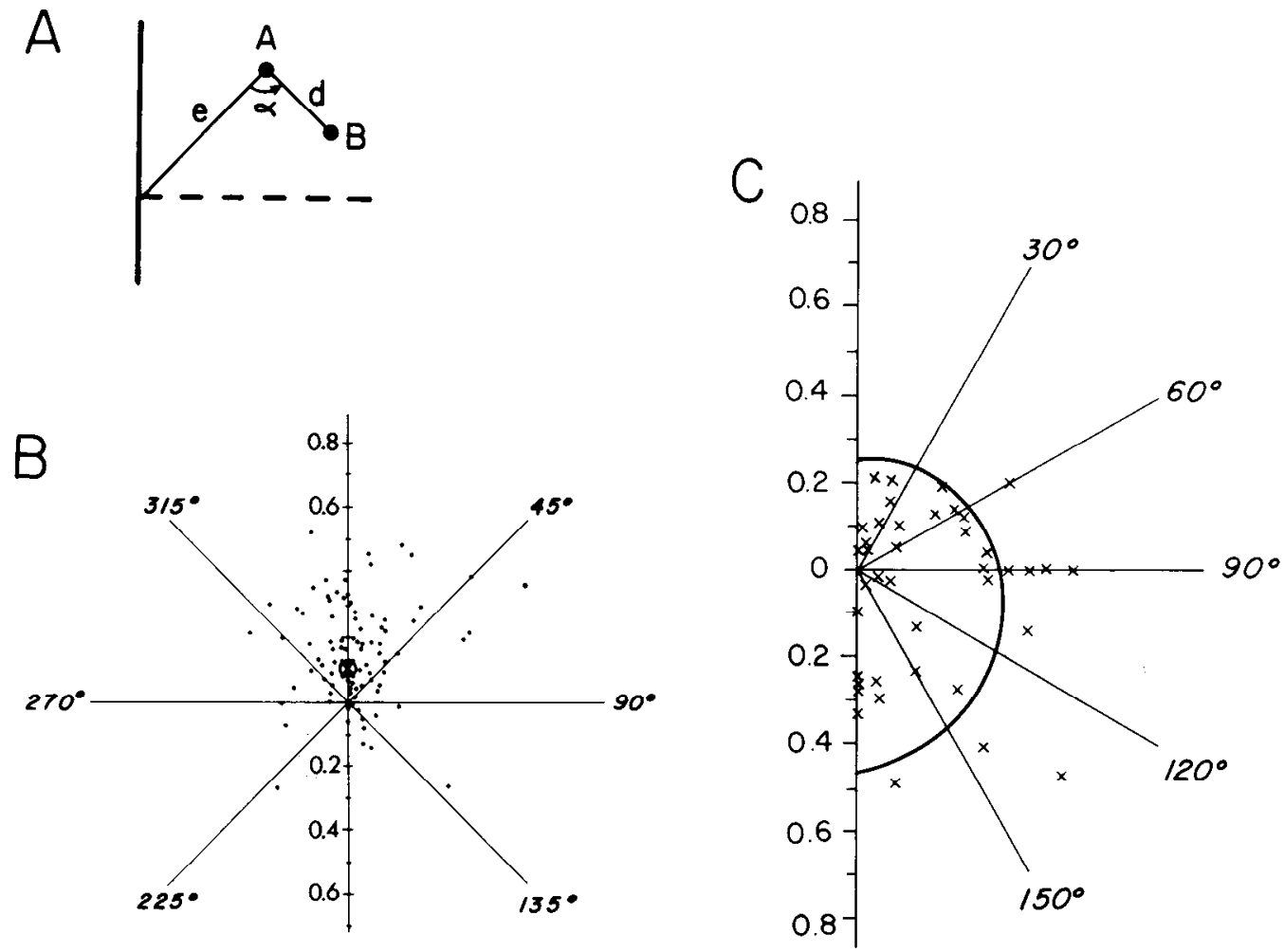

Figure 11. A, Procedure for plotting spatial relation of pairs of visual field points. $A$ is a point in the visual field at eccentricity $e . B$ is a second point in the visual field at a distance $d$ from point $A$. The angle $\alpha$ is the angle formed by line segments $d$ and $e$ (measured counterclockwise). The spatial relationship of the two points may be described in polar coordinates as a single point of coordinates $r, \alpha$, where $r=d / e$, and is a measure of the separation of points $A$ and $B$ scaled for eccentricity. $B$, Distribution of activity centers relative to geometric centers for multiunit fields. Each point is plotted by the procedure described above; $A$ represents the geometric center and $B$ the activity center of a multiunit field. All points are from a single hemisphere. The $X$ near the center represents the average expected position of the activity center as calculated in Appendix $B$. $C$, Distribution of single unit field centers relative to multiunit field centers as plotted by the same procedure. $A$ now represents the activity center of the multiunit field, and $B$ represents the center of a single unit receptive field. Additionally, points for which $\alpha>180^{\circ}$ have been reflected about the line passing through point $A$ and the origin. The solid curve shows the distribution of the centers of single unit receptive fields at each eccentricity whose edges would just include the activity center of the multiunit field, assuming the approximation: receptive field radius $=0.32 \times$ eccentricity. Multiunit fields near the fovea have been excluded to minimize the distortion inherent in this approximation. 

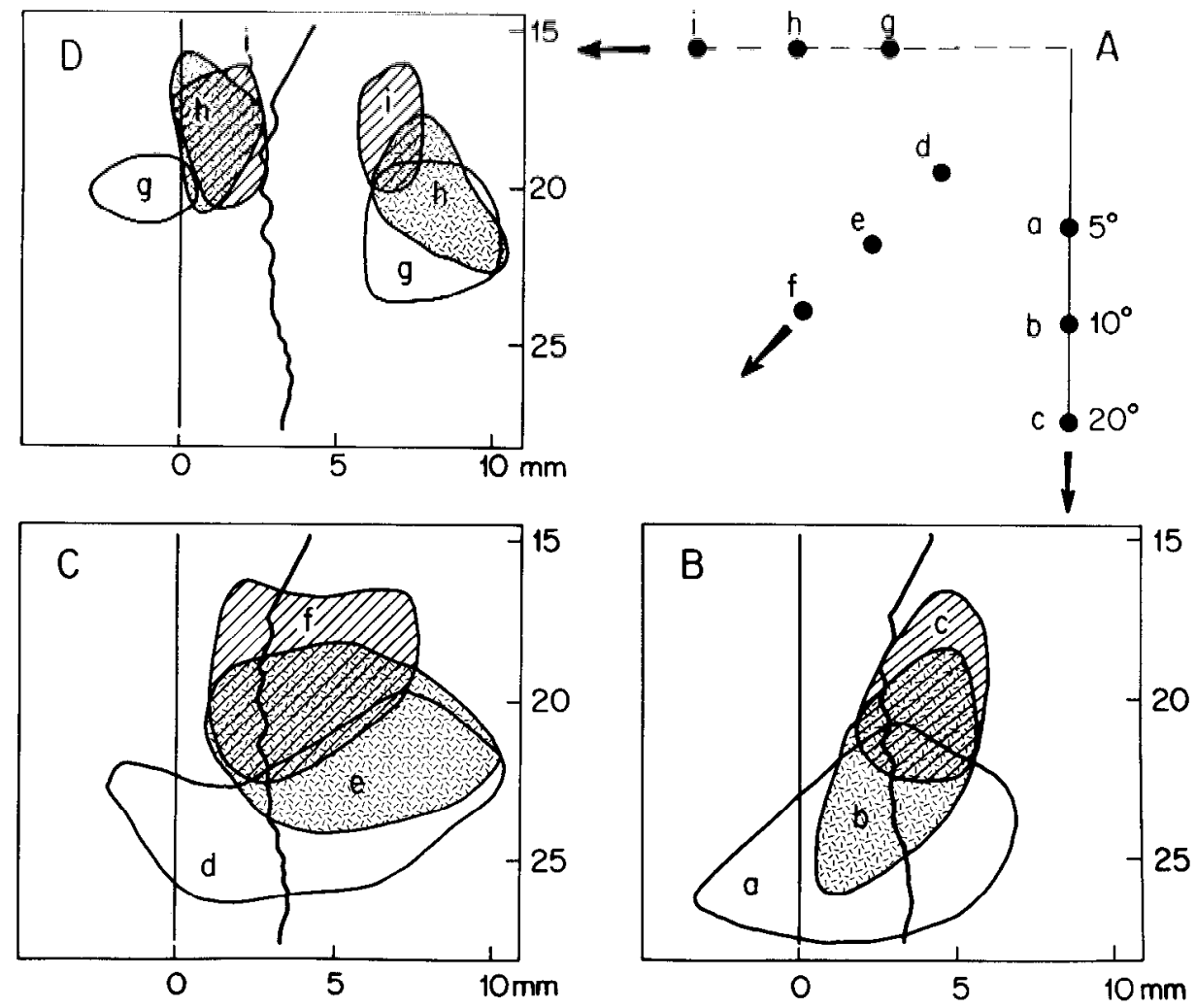

Figure 12. Cortical point images for the nine visual field points shown in $A$. $B$, Point images for points along the vertical meridian at $5^{\circ}, 10^{\circ}$, and $20^{\circ}$ eccentricity. $C$, Point images for points along the $45^{\circ}$ meridian at the same eccentricities. $D$, Point images for points along the horizontal meridian at the same eccentricities.

activity center that would allow the single unit field to still contain the activity center. The curve in Figure $11 C$ shows the locus of all such points. Figure $11 C$ also shows the actual distribution of single unit field centers relative to multiunit field activity centers. Most points lie within the curve, a result again consistent with the idea that there is topographic regularity in $\mathrm{AL}$ and $\mathrm{PM}$.

\section{Point images and topography}

In studying receptive fields of visual neurons, one asks how much of the visual field is responded to by a single cell. Another way to analyze visual topography is to start with a single point in the visual field and ask how many cells respond to that point. A cortical "point image" is that area of the cortex functionally connected to a point on the retina (McIlwain, 1976). In a topographically organized region, point images for separated points in the visual field should be displaced on the cortex.

Figure 12 shows the point images for nine retinal points in the hemisphere where we had the most complete data. Each point image contains all neighboring recording sites whose multiunit fields overlapped the appropriate retinal point. The point images are quite large, and there is a clear tendency for point image size to increase for smaller eccentricities. Although there is considerable overlap, point images for different retinal points are clearly displaced in the direction to be expected from the topographic summary maps. Further- more, the sizes and locations of the point images are consistent with the presence of two representations of the lower quadrant. There are two clearly separated sets of point images for points along the horizontal meridian and a single set of images for points along the vertical meridian. There is a single set of images for $45^{\circ}$ meridian points, and these images are almost twice the size of the horizontal and vertical meridian images; this result is consistent with the presence of two displaced representations of that meridian, one in PM and a second in AL.

\section{Discussion}

We have described topographic organization in a large region of extrastriate cortex occupying the prelunate gyrus and extending into the anterior bank of the lunate sulcus and the posterior bank of the superior temporal sulcus. Our data suggest that much of this cortex is occupied by two orderly, mirror-image representations of the quadrant, PM and AL, which share a common representation of the vertical meridian. Since we have mapped both AL and PM and determined their relation to each other in only one hemisphere, it is valid to ask how consistent this visuotopic organization is likely to be from one hemisphere to another. The strongest evidence in favor of subdividing this cortex is the presence in all hemispheres of a representation of the vertical meridian. We mapped the anterolateral area in three hemispheres, and the posteromedial area in two, and 


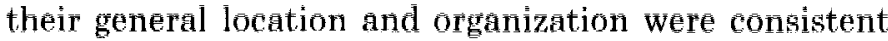
with the scheme derived from the best-studied hemisphere. Our data suggest that the location of the vertical meridian representation, and consequently of the two areas, relative to brain landmarks will probably vary from hemisphere to hemisphere. Van Essen et al. (1981) have also described variation in size, shape, and precise geographic location of another visual area, area MT, in the macaque monkey.

Our data suggest that the representation of the visual field in PM and AL is limited to about the central $30^{\circ}$ of vision and does not include the periphery. We did not find activity centers peripheral to $30^{\circ}$. In MT, Gattass and Gross (1981) found that field centers at $55^{\circ}$ eccentricity were sufficient to permit representation of the whole of the visual field. A little more than $2 \mathrm{~mm}$ of cortex along the meridia in AL and PM would contain those centers if magnification factor calculations are extrapolated into the periphery. It is thus unlikely that all such sites would have been missed in our recordings. The absence of peripheral field representation in $\mathrm{AL}$ and PM is in agreement with a recent report of Ungerleider et al. (1983), who found an absence of projections to V4 from parts of $\mathrm{V} 2$ representing the visual field peripheral to $40^{\circ}$.

We have determined the boundries of areas $\mathrm{AL}$ and PM along the parafoveal and peripheral horizontal meridian representations. Dorsally, PM is bordered by a zone of cortex with qualitatively different properties. The posterior part of this zone had recording sites in the upper visual quadrant, the anterior part had recording sites with receptive fields in the lower quadrant. However, in neither part of that zone did our techniques reveal the same degree of topographic order as in AL and PM. Furthermore, receptive fields in the anterior part were larger than in AL or PM. Anteriorly, AL is bordered by a narrow strip of cortex which separates it from MT. Ungerleider et al. (1982) reported that portions of MT representing the lower visual field and fovea project to narrow zone of cortex just posterior to and bordering MT. This anatomically defined region may correspond to the transition cortex between $\mathrm{AL}$ and $\mathrm{MT}$ which we have defined physiologically.

\section{Are areas $A L$ and PM part of the V4 complex?}

The areas we have described occupy much of the prelunate gyrus and extend into the lunate and superior temporal sulci. The V4 complex is shown to occupy the lateral part of the gyrus and to extend into both sulci (Zeki, 1971, 1977, 1978a, b; Van Essen and Zeki, 1978).

There is, thus, considerable overlap between the cortex we have explored and the cortex of the V4 complex. However, it is not yet possible to decide if our physiologically defined regions should be properly assigned to the V4 complex, an anatomically defined region. Zeki (1971) defined V4 as a broad region receiving projections from $\mathrm{V} 2$ and V3. It is thus necessary to know the full extent of the projections from V2, especially from areas of peripheral representation, in order to know the full extent of V4. These data are not yet available since it is only recently that such a study has been undertaken
(Ungerleider et àl, 1983). Vāñ Esseñ and Zeki (1978) suggested that, dorsally, V4 is bordered by V3A, but they did not determine the precise location of this border. It is probable that the anterolateral area, $A L$, is part of the V4 complex. PM may be part of the V4 complex, but it might instead be part of VBA, or even an independent area associated with neither.

We have proposed that AL and PM form major functional subdivisions of the prelunate gyrus and may be part of the V4 complex. This idea is consistent with the repeatedly made suggestion that the $\mathrm{V} 4$ complex consists of several subdivisions (Zeki, 1971, 1977; Van Essen and Zeki, 1978; Felleman and Van Essen, 1983). The earlier anatomical studies (Zeki, 1971) distinguished between $\mathrm{V} 4$, in the anterior bank of the lunate, and V4A, on the prelunate gyrus. There is thus some correspondence between V4 and PM, and V4A and AL, since PM is buried in the lunate sulcus and AL occupies the gyrus. However, our results show PM posteromedial to AL, whereas Zeki (1971) shows V4 and V4A adjacent in the same horizontal sections at relatively lateral levels. It is difficult to make a final judgment about the proper assignment of $\mathrm{AL}$ and PM without more complete anatomical data or experiments which compare the entire projection zone of V2 with the location of these physiologically defined areas.

Medial to the horizontal meridian representation on the gyrus in PM we found sites with fields in the upper quadrant. Conceivably, this region is a continuation of area PM so that the map in PM could be described as a first-order transformation. Alternatively, this region might correspond to the representation of the upper visual field in V3A (Van Essen and Zeki, 1978; Zeki, $1978 \mathrm{a})$. It is also possible that there are actually two rather small representations of the upper visual field, associated, respectively, with V3A and PM; this might well lead to the apparent disorder in the retinotopic organization we saw. We have also been unable to assign the anterior part of this dorsal bordering cortex. By Van Essen and Zeki's (1978) summary scheme, it could either be part of V3A, a part of the V4 complex, or yet another subdivision. However, it is well dorsal to the part of V4 examined by Van Essen and Zeki (1978). Furthermore, it lies mainly on the surface, whereas the part of V3A that Van Essen and Zeki (1978) explored lies in buried cortex.

\section{Comparison with other studies of prelunate gyrus topography}

Several studies have looked at topography of the prelunate gyrus using anatomical and physiological techniques. The physiological data are from rather small extents of this cortex; the whole region has not previously been mapped systematically.

Fischer et al. (1981) recorded from the prelunate gyrus a few millimeters above the tip of the inferior occipital sulcus. The majority of their recording sites would fall well within our area AL. Multiunit field centers were found in the central $5^{\circ}$ of the visual field. They concluded that the area was not retinotopically organized, but they based this conclusion upon a scatter plot which plotted distance of receptive fields in degrees as a function of 
distance between recording sites in millimeters. The resulting plot appeared random compared to the corresponding plot for a section of striate cortex. This is not, however, a very sensitive way to compare the degree of retinotopic organization, since it does not take into account the differences in magnification factor in the two regions. We have found magnification factor in $\mathrm{AL}$ to be considerably smaller than in V1.

Zeki (1977) shows a number of penetrations into the superior temporal sulcus lateral to MT. Most cells had receptive fields located along the parafoveal horizontal meridian. He noted an orderly movement of receptive fields in this area and concluded that it was topographic. These recordings are consistent with our finding of a horizontal meridian representation within $\mathrm{AL}$ a few millimeters below the lip of the superior temporal sulcus. Van Essen and Zeki (1978) recorded from sites on the prelunate gyrus, the anterior bank of the lunate sulcus, and the posterior bank of superior temporal sulcus. They argued that this region must have some degree of topographic order, since they found orderly shifts in receptive field position on penetrations parallel to the surface, and not on perpendicular penetrations. However, they also found distant recording sites with nearby receptive ficlds, and large shifts in field positions for nearby recording sites, suggesting that organization was more complex than in a simple representation of the quadrant. These recordings were primarily from sites with receptive fields within the central $5^{\circ}$ of vision, the region where topography will be most difficult to determine. The area explored, then, overlaps the area we studied, but our recordings extended more dorsally, into regions of parafoveal and peripheral representation, where topography is considerably easier to see. Thus the data of Van Essen and Zeki (1978) are not inconsistent with the organization we propose.

Inferences about topography of the prelunate gyrus have also been drawn from anatomical studies. However, these must be interpreted with caution, especially for regions like $\mathrm{AL}$ and $\mathrm{PM}$. We have shown that point images in $\mathrm{AL}$ and $\mathrm{PM}$ are very large. This means that information from a single retinal point is spread over a relatively large proportion of the entire area. The point image data suggest that anatomical experiments could not clearly resolve even the presence of two areas unless projections from regions of horizontal meridian representation were compared to projections from vertical meridian representation. Projections from more closely spaced sites could hardly he expected to suggest the degree of topographic order we have seen physiologically.

Van Essen and Zeki (Zeki, 1969, 1970; Van Essen et al., 1982) have suggested that the distribution of degeneration within the V4 complex following callosal section may be used to define the region of vertical meridian representation and thus the boundaries of V4. At least some callosal fibers carry information about the vertical meridian (Hubel and Wiesel, 1967), and Van Essen and Zeki (1978) found that receptive fields of cells in areas of dense degeneration were close to the vertical meridian, although there were numerous exceptions to this pattern. The callosal degeneration in V4 is complex and patchy, thus suggesting a far less organized vertical meridian representation than we have seen physiologically. However, this discrepancy is probably more apparent than real. Not all callosal fibers are concerned with the meridian, nor are they the only, or even the most important, source of such information (Rocha-Miranda et al., 1975; Gross et al., 1977). Furthermore, in other visual areas the vertical meridian representation does not correspond well with the pattern of callosal degeneration. In MT, degeneration following callosal section is widespread and not restricted to the border of MT where the vertical meridian is represented (Gattass and Gross, 1981; Van Essen et al., 1981). In the owl monkey, the pattern of degeneration is complex over several of the dorsal third tier areas (Newsome and Allman, 1980) and shows no striking correlation with the vertical meridian representation. Therefore, it does not seem that data on callosal projections to V4 are incompatible with the topographic organization we describe.

Zeki (1971) looked at the pattern of degeneration seen after lesions of V2 affecting central visual fields. Degeneration was seen on the prelunate gyrus at and just above the level of the inferior occipital sulcus. This is in agreement with our results showing that central vision is represented laterally on the gyrus. Zeki $(1978 \mathrm{c})$ also found a direct projection from the central $1^{\circ}$ of the representation in $\mathrm{V} 1$ to the more lateral and posterior part of V4. This is again in agreement with our placement of the foveal representation in AL and PM. It should be noted, however, that a more recent study (Felleman and Van Essen, 1983) failed to confirm the finding of projections from V1 to V4.

\section{Is there an expanded representation of the fovea in $A L$ and $P M$ ?}

$\mathrm{PM}$ and $\mathrm{AL}$ contain a representation of only the central $30^{\circ}$ of the visual field. Zeki (1971, 1977; Van Essen and Zeki, 1978) has suggested that V4 might be specialized for central vision and that the fovea might be overrepresented here compared to other visual areas. Our analyses suggests that there is an overrepresentation of the fovea in $\mathrm{AL}$ and PM, but that this is not unique.

Comparison of point image sizes for retinal points at different eccentricities can show the relative cortical representations of different parts of the visual field. If the same volume of cortex is functionally connected to each point in the visual field, point images should be the same size, or "translationally invariant" across the visual field. When this is true, the fovea is not overrepresented. We determined point images directly for areas AL and PM and found a clear general tendency for sizes of point images to increase at smaller eccentricities. Thus, point images on the prelunate gyrus are not transitionally invariant, and there is an overrepresentation of the fovea.

Is the overrepresentation of the foveal field in $\mathrm{AL}$ and PM also present in other cortical areas? Point images have not been measured directly in other areas, but one can estimate point image size as the product of magnification factor and multiunit field size.

There are reports implying translational invariance in striate cortex in both cats and monkeys (Albus, 1975; 
Hubel and Wiesel, 1974). However, a recent paper by Dow et al. (1981) suggested that point imanges derrived from magnification factor and aggregate field size were much larger in the fovea than in the periphery. Gross and collaborators (Gattass and Gross, 1981; Gattass et al., 1981) have published data on magnification factor and multiunit field size for $\mathrm{V} 1, \mathrm{~V} 2$, and $\mathrm{MT}$ which imply larger point images in the fovea than in the periphery for all three areas. In order to compare their studies with our own, we have calculated point image size as a function of eccentricity (Fig. 13), using curves describing magnification factor and multiunit field size as a function of eccentricity, for AL, MT, V2, and V1. In all cases, the magnification factor curve was the best fitting power function and the field size curve was the linear regression line. Figure 13 shows that the overrepresentation of small eccentricities in AL is also present to a similar extent in $\mathrm{V} 1, \mathrm{~V} 2$, and MT.

\section{Limitations to the accuracy of determining topography in foveal and parafoveal representations}

Proper placement of horizontal and vertical meridian representations is extremely important in determining topography, since the meridia have repeatedly been shown to form boundaries between different visual areas. We suggest that there are intrinsic limitations in the electrophysiological determination of these boundaries in regions of foveal and parafoveal representation where receptive fields are large. Estimates of these boundaries will be poorer at foveal than at peripheral locations, since foveal fields may overlap both meridia and therefore will be difficult to assign to either. This problem becomes particularly severe for AL and PM, since receptive field size does not become vanishingly small at the central fovea. When foveal fields are relatively large, measurement error in determining activity centers could easily cause them to be assigned to the incorrect meridian. There are many such sources of measurement error. Misplacing the true activity center of a multiunit receptive field is an inevitable consequence of sampling the activity of only a few of the many cells in the cortical column. Other factors such as variability in responsiveness, slight variations in fixation on different trials, and

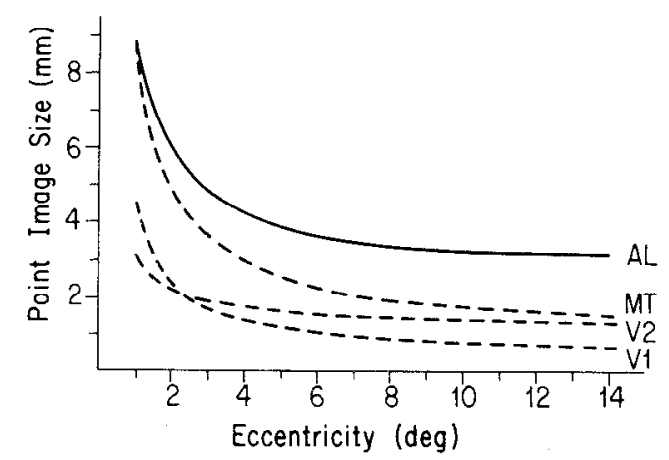

Figure 13. Point image size as a function of eccentricity in V1, V2, MT, and AL, calculated as the product of magnification factor and multiunit field size. Data for AL are from the present paper. Data for MT are from Gattass and Gross (1981); data for V1 and V2 are from Gattass et al. (1981).

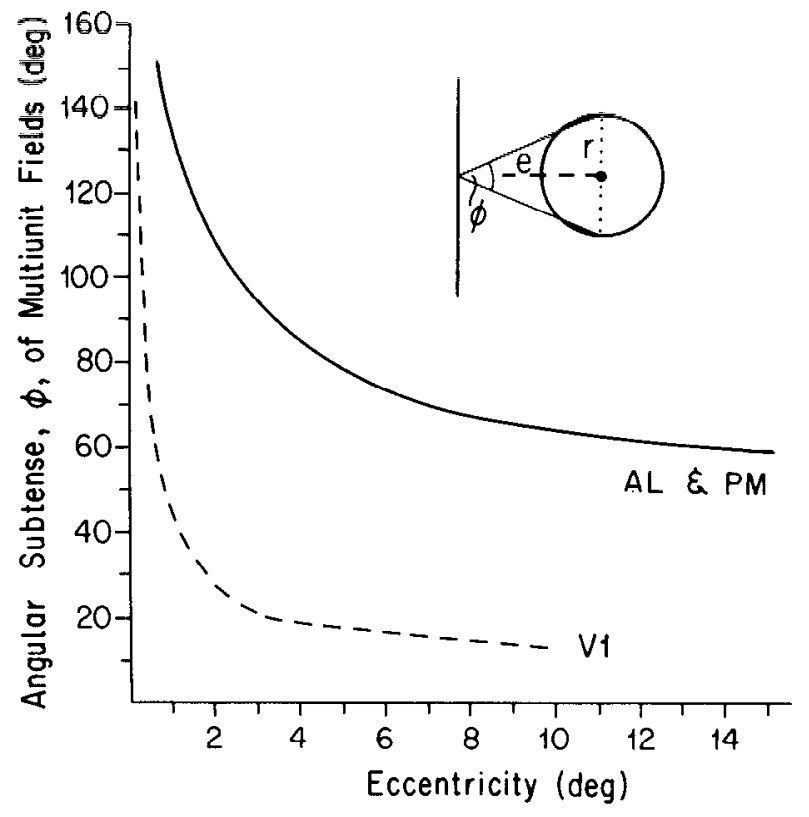

Figure 14. Angular subtense of multiunit fields in AL and PM compared with V1. The curve for $A L$ and $P M$ is based on data from this report. The $V 1$ curve is derived from the data of Gattass et al. (1981). The inset shows how we define angular subtense. Multiunit fields are assumed to be circular, with radius $r=\sqrt{\frac{\text { area }}{\pi}}$. For a multiunit field with its center at eccentricity $e$, angular subtense, $\phi,=2 \times \arctan (r / e)$.

different recording properties of different electrodes will also contribute. However, an upper bound on measurement error is set by the size of the multiunit fields. Thus, we propose that the uncertainty in the estimate of the proper angular visual field coordinate of a multiunit field will be proportional to the angular "subtense" of that multiunit field; that is, the angle formed between the lateral borders of the field and the center of the visual field (Fig. 14, inset). Fields with an angular subtense of $90^{\circ}$ or greater will always include both horizontal and vertical meridia. Fields with an angular subtense between $70.5^{\circ}$ and $90^{\circ}$ will always include at least one meridian and may include both. We have calculated the angular subtense as a function of eccentricity for typical multiunit fields in AL and PM and, for comparison, for multiunit fields in V1 using the data of Gattass et al. (1981). It is clear from Figure 14 that when angular subtense is used as a measure of the precision with which meridional boundaries can be located, the boundaries of $\mathrm{AL}$ and PM must be far less certain than those of V1. While the uncertainty is already considerable at parafoveal locations, it becomes far greater within the central $3^{\circ}$.

\section{Topographic irregularity in $A L$ and $P M$}

We have analyzed topography by constructing visuotopic summary maps from sector maps. 'This technique could certainly allow one to overlook small discontinuities in the representation, and even rather substantial partial representations of the visual field as long as they were contained within a reasonably small area of the 
cortex. Although this is clearly a shortcoming to this quasistatistical approach, we nonetheless felt that this approach was successful in revealing topographic order in $\mathrm{AL}$ and PM. It is possible to argue that the overlap of sector representations and the occasional disparate points and reversals in the progression of receptive field trajectories (e.g., Fig. 6, penetration b) suggest topographic irregularity. However, we did nol see consistent irregularities in the representation from hemisphere to hemisphere. The irregularities we did see might be due simply to measurement error. They might also be real and difficult to see in comparison from animal to animal, again because of the problem of measurement error. It is particularly difficult to decide between these alternatives in areas where multiunit fields are quite large, and a large proportion of cells are concerned with visual field locations close to the fovea. We conclude that the best interpretation of our results is that topography in PM and $\mathrm{AL}$ is qualitatively similar to topography seen at earlier stages in the primate visual system.

\section{Appendix A}

The standard regression line $Y=a x+b$ is chosen so that

$$
\sum_{j=1}^{n}\left(y_{j}-\left(a x_{j}+b\right)\right)^{2}
$$

is a minimum. We wish to choose the line $a x+b$ such that

$$
\sum_{j=1}^{n}\left(\frac{y_{j}-a x_{j}-b}{x_{j}}\right)^{2}
$$

is a minimum. This is equivalent to

$$
\sum_{j=1}^{n}\left(\frac{y_{j}}{x_{j}}-\frac{b}{x_{j}}-a\right)^{2}=\sum_{j=1}^{n}\left(\frac{y_{j}}{x_{j}}-\left(\frac{b}{x_{j}}+a\right)\right)^{2}
$$

which is the standard regression line for a variable

$$
y^{\prime}=\frac{y_{j}}{x_{j}} \text { as a function of variable } x^{\prime}=\frac{1}{x_{j}}
$$

The slope of this computed regression line (obtained by dividing all size values by their corresponding eccentricity, inverting all eccentricities, and feeding these data to a regression program) is equivalent to the intercept of the function we desire. Likewise, the intercept of this function gives one the slope of the desired function. That is:

$$
y^{\prime}=b x^{-1}+a
$$

and we desire

$$
y=a x+b
$$

This function will give very good estimates of the true relationship in the data with one rather obvious proviso. Use of fields on or very close to $0^{\circ}$ eccentricity will distort the function unless a small correction is made for the fact that the scatter in field size is non-zero at $0^{\circ}$ eccentricity.

\section{Appendix B}

We can predict the expected relation between activity centers and geometric centers. We assume that the ac- tivity center of the multiunit field is the best estimate of the point represented by that field. To compare data from different eccentricities, we must scale distances according to the equation describing receptive field size as a function of eccentricity. The activity center is at a point $E_{\mathrm{s}}$, the geometric center is at a point $E_{\mathrm{g}}$.

The activity center then will lie between two points call $E_{\text {near }}$ and $E_{\text {far. }}$. These are points which lie a receptive field radius distant from the activity center toward $\left(\bar{E}_{\text {near }}\right)$ and away $\left(E_{\text {far }}\right)$ from the fovea, respectively. Thus, using our single unit receptive field size regression curve,

$$
\begin{gathered}
E_{\mathrm{n}}+0 . \overline{3} \overline{2} \bar{E}_{\mathrm{n}}+1.01-E_{\mathrm{n}} \\
E_{\mathrm{f}}-0.32 \bar{E}_{\mathrm{f}}-1.01=E_{\mathrm{g}}
\end{gathered}
$$

The geometric center will lie at the point midway between the two, thus

$$
E_{\mathrm{g}}=\frac{E_{\mathrm{n}}+E_{\mathrm{f}}}{2}
$$

Simple algebra yields the equation

$$
E_{\mathrm{g}}=1.11 E_{\mathrm{a}}+0.36
$$

If we ignore the constant, significant only at small eccentricities,

$$
E_{\mathrm{a}}=0.9 E_{\mathrm{g}} \text { in } \mathrm{PM} \text { and } \mathrm{AL}
$$

\section{References}

Albus, K. (1975) A quantitative study of the projection area of the central and paracentral visual field in area 17 of the cat. I. The precision of the topography. Exp. Brain Res. 24: 159179.

Allman, J. M., and J. H. Kaas (1971) A representation of the visual field in the caudal third of the middle temporal gyrus of the owl monkey (Aotus trivirgatus). Brain Res. 31: 85-105.

Allman, J. M., and J. H. Kaas (1974a) The organization of the second visual area (VII) in the owl monkey: A second order transformation of the visual hemifield. Brain Res. 76: 247265.

Allman, J. M., and J. H. Kaas (1974b) A crescent-shaped cortical visual area surrounding the middle temporal area (MT) in the owl monkey (Aotus trivirgatus). Brain Res. 81: 199-213.

Allman, J. M., and J. H. Kaas (1975) The dorsomedial cortical visual area: A third tier area in the occipital lobe of the owl monkey (Aolus trivirgalus). Brain Res. 100: 473-487.

Allman, J. M., and J. H. Kaas (1976) Representation of the visual field on the medial wall of the occipital-parietal cortex in the owl monkey. Science 191: 572-575.

Baizer, J. S. (1982) Receptive field properties of V3 neurons in monkey. Invest Ophthalmol. 23: 87-95.

Baizer, J. S., and W. M. Maguire (1983) Double representation of lower visual quadrant in prelunate gyrus of rhesus monkey. Invest. Ophthalmol. 24: 1436-1439.

Baizer, J. S., D. L. Robinson, and B. M. Dow (1977) Visual responses of area 18 neurons in awake, behaving monkey. J. Neurophysiol. 40: 1024-1037.

Baker, J. F., S. E. Petersen, W. T. Newsome, and J. M. Allman (1981) Visual response properties of neurons in four extrastriate visual areas of the owl monkey (Aotus trivirgatus): A quantitative comparison of medial, dorsomedial, dorsolateral, and middle temporal areas. J. Neurophysiol. 45: 397-416.

Dow, B. M., A. Z. Snyder, R. G. Vautin, and R. Bauer (1981) Magnification factor and receptive field size in foveal striate cortex of the monkey. Exp. Brain Res. 44: 213-228.

Felleman, D. J., and D. C. Van Essen (1983) The connections 
of area V4 of macaque monkey extrastriate cortex. Soc. Neurosei. Abstr. 9: 153.

Fischer, B., R. Boch, and M. Bach (1981) Stimulus versus eye movenents: Comparison of neural activity in the striate and prelunate visual cortex (A17 and A19) of trained rhesus monkey. Exp. Braiñ Res. 43: 69-77.

Gattass, R., and C. G. Gross (1981) Visual topography of striate projection zone (MT) in posterior superior temporal sulcus of the macaque. J. Neurophysiol 46: 621-638.

Gattass, R., C. G. Gross, and J. H. Sandell (1981) Visual topography of V2 in the macaque. J. Comp. Neurol. 201: 519540 .

Gross, C. G., D. B. Bender, and M. Mishkin (1977) Contributions of the corpus callosum and the anterior commissure to visual activation of inferior temporal neurons. Brain Res. 131: 227-239.

Hubel, D. H., and T. N. Wiesel (1967) Cortical and callosal connections concerned with the vertical meridian of the visual fields in the cat. J. Neurophysiol. 30: 1561-1573.

Hubel, D. H., and T. N. Wiesel (1972) Laminar and columnar distribution of geniculocortical fibers in the macaque monkey. J. Comp. Neurol. 146: 421-450.

Hubel, D. H., and T. N. Wiesel (1974) Uniformity of monkey striate cortex: A parallel relationship between field size, scatter, and magnification factor. J. Comp. Neurol., 158: 307318.

Mcllwain, J. 'l'. (1976) Large receptive fields and spatial transformations in the visual system. Int. Rev. Physiol. 10: 223248.

Maguire, W. M., and J. S. Baizer (1981) Visuotopic organization on the prelunate gyrus of the rhesus monkey. Soc. Neurosci. Abstr. 7: 172.

Maguire, W. M., and J. S. Baizer (1982) Luminance coding of briefly presented stimuli in area 17 of the rhesus monkey. J. Neurophysiol. 47: 128-137.

Newsome, W. T., and J. M. Allman (1980) Interhemispheric connections of visual cortex in the owl monkey (Aotus trivirgatus), and the bushbaby (Galago senegalensis). J. Comp. Neurol. 194: 209-233.

Rocha-Miranda, C., D. B. Bender, C. G. Gross, and M. Mishkin (1975) Visual activation of neurons in inferotemporal cortex depends on striate cortex and the forebrain commissures. J. Neurophysiol. 38: 475-491.
Ungerleider, L. F., R. Desimone, and M. Mishkin (1982) Cortical projections of area MT in the macaque. Soc. Neurondi. Abstr. 8: 680 .

Ungerleider, L. G., R. Galtass, and A. P. B. Sousa (1989) Projections of area V 2 in the macaque, Soc. Neurosci. Abstr. 9: 152 .

Van Essen, D. C. (1979) Visual areas of the mammalian cerebral cortex. Annu. Rev. Neurosci. 2: 227-263.

Van Essen, D. C., and J. H. R. Maunsell (1980) Two-dimensional maps of the cerebral cortex. J. Comp. Neurol. 191: $255-281$.

Van Essen, D. C., and S. M. Zeki (1978) The topographic organization of rhesus monkey prestriate cortex. J. Physiol. (Lond.) 277: 193-226.

Van Essen, D. C., J. H. R. Maunsell, and J. L. Bixby (1981) The middle temporal visual area in the macaque: Myeloarchitecture, connections, functional properties, and topographic organization. J. Comp. Neurol. 199: 293-326.

Van Essen, D. C., W. T. Newsome, and J. L. Bixby (1982) The pattern of interhemispheric connections and its relationship to extrastriate visual areas in the macaque monkey. J. Neurosci. 2: 265-283.

Wurtz, R. H. (1969) Visual receptive fields of striate cortex neurons in awake monkeys. J. Neurophysiol. 32: 727-742.

Zeki, S. M. (1969) Representation of central visual fields in prestriate cortex of monkey. Brain Res. 14: 271-291.

Zeki, S. M. (1970) Interhemispheric connections of prestriate cortex in the monkey. Brain Res. 19: 63-75.

Zeki, S. M. (1971) Cortical projections from two prestriate areas in the monkey. Brain Res. 34: 19-35.

Zeki, S. M. (1973) Colour coding in rhesus monkey prestriate cortex. Brain Res. 53: 422-427.

Zeki, S. M. (1977) Colour coding in the superior temporal sulcus of rhesus monkey visual cortex. Proc. R. Soc. Lond. (Biol.) 197: 195-223.

Zeki, S. M. (1978a) The third visual complex of rhesus monkey prestriate cortex. J. Physiol. (Lond.) 277: 245-272.

Zeki, S. M. (1978b) Uniformity and diversity of structure and function of rhesus monkey prestriate visual cortex. J. Physiol. (Lond.) 277: 273-290.

Zeki, S. M. (1978c) The cortical projections of foveal striate cortex in the rhesus monkey. J. Physiol. (Lond.) 277: 227244 . 